DOI: https://doi.org/10.11144/Javeriana.upsy16-2.avpm

\title{
Adaptación y validación en población mexicana del Cuestionario de personalidad tipo C (PCTC)*
}

\section{Adaptation and validation of the Mexican population type $\mathrm{C}$ personality questionnaire (PCTC)}

Recepción: 08 Abril 2014 | Aprobación: 24 Enero 2017

\author{
Marina Ceballos Fontes \\ Universidad Veracruzana, México \\ María José García Oramas \\ Universidad Veracruzana, México \\ Roberto Lagunes Córdoba ${ }^{\mathrm{a}}$ \\ Universidad Veracruzana, México \\ ORCID: http://orcid.org/0000-0003-0650-4085
}

a Autor de correspondencia. Correo electrónico: rlc.academico@yahoo.com.mx

Para citar este artículo: Ceballos, M., García, M.J., Lagunes, R. (2017). Adaptación y validación en población mexicana del Cuestionario de personalidad tipo C (PCTC). Universitas Psychologica 16(2), 1-11 https://doi.org/10.11144/Javeriana.upsy16-2.avpm

\section{RESUMEN}

El objetivo de este trabajo es la adaptación y validación del Cuestionario de personalidad tipo C, elaborado por López, Ramírez y Esteve \& Anarte $(1998,2002)$ a una población mexicana. Se realizó una adaptación lingüística del Cuestionario, y se aplicó a una muestra de 391 personas en las ciudades de Chihuahua y Xalapa, México. El análisis factorial llevó a eliminar seis de los reactivos de la escala original. El resto de los reactivos se agrupan en cuatro factores. El análisis factorial confirmatorio indica que los factores fundamentales del constructo de personalidad tipo $\mathrm{C}$ en población mexicana son los de comprensión, no expresión emocional y necesidad de armonía, aunque en la muestra de la ciudad de Xalapa, este último factor no fue fundamental. La confiabilidad total de la escala por el método alfa de Cronbach fue de 0.757 , y en las cuatro subescalas osciló entre 0.645 y 0.842 .

Palabras clave

personalidad tipo C; escala; adaptación; validación

\begin{abstract}
The aim of this work was the adaptation and validation of the TypeC Personality scale C, by Lopez, Ramirez and Esteve \& Anarte (1998, 2002). Linguistic adaptation of the questionnaire was carried out with a sample of 391 people in the cities of Chihuahua and Xalapa, México. Factor analysis led to eliminate six items from the original scale. The remaining items were grouped in four factors. Confirmatory factor analysis indicates that the fundamental factors of type $\mathrm{C}$ personality construct are understanding, emotional inexpressiveness, and need for harmony. Nevertheless, need for harmony was not fundamental in the sample from Xalapa. The overall reliability (Cronbach's alpha) of the scale was 757 and for the four subscales ranged from 0.645 to 0.842 .

Keywords

type-C personality; scale; adaptation; validation
\end{abstract}




\section{Introducción}

La relación entre diferentes tipos de personalidad y las enfermedades psíquicas han sido una preocupación constante en la psicología de la salud. A lo largo del tiempo, se ha propuesto que ciertos rasgos psicológicos y emocionales se asocian con el surgimiento y agravamiento de diversas enfermedades (Amaya \& Parra, 2010; Sebastián, León, \& Hospital, 2009). En el contexto particular del estudio del cáncer de mama, se ha propuesto la existencia de un tipo de personalidad relacionado a factores de riesgo y estilos de afrontamiento de la enfermedad: la personalidad tipo C (Morris \& Greer, 1980).

La personalidad tipo $\mathrm{C}$ se ha definido como un estilo de comportamiento que se caracteriza por la supresión de emociones ante situaciones estresantes (Anarte, López, Ramírez \& Zarazaga, 2000). La supresión emocional constituye el aspecto central de este patrón de conducta, y se considera una respuesta consciente de autocontrol de sentimientos negativos (López, Esteve, Ramírez, \& Anarte, 1998; Morris \& Greer, 1980).

La supresión o control de sentimientos negativos y hostiles, como el enojo, se lleva a cabo a través de un comportamiento racional, mediante el cual la persona intenta comprender y tratar bien a los demás. Este aspecto se traduce en una tendencia a mantener relaciones interpersonales armoniosas, incluso cuando los demás actúan en contra de los intereses de la persona (López, Ramírez, Esteve \& Anarte, 2002).

Quienes han desarrollado la personalidad tipo C suelen presentar las siguientes características: son personas excesivamente serviciales, pacificadoras, presentan dificultad para defender sus derechos, son pacientes, sumisas, evitan conflictos, buscan la armonía en las relaciones interpersonales, suprimen la expresión de las emociones y son incapaces de enfrentarse a tensiones en el ámbito interpersonal, lo cual les lleva a experimentar sentimientos de indefensión y desesperanza, que además las hace propensas a desarrollar trastornos de depresión (Anarte et al., 2000;
Durá, Pérez, Ferrando, Murgui \& Martínez, 2010; López et al., 1998, 2002).

La personalidad tipo $\mathrm{C}$ es un constructo complejo, cuya estructura ha sido objeto de polémica en diferentes estudios. El análisis llevado a cabo por López et al. (1998) sobre instrumentos construidos para medir diferentes aspectos de este tipo de personalidad, permitió concluir que existen cinco factores que la caracterizan:

Control emocional: se refiere a que las personas usan la razón y la lógica para evitar expresar emociones socialmente negativas.

Racionalidad:es una forma de utilizar la razón y la lógica para enfrentarse a situaciones de la vida diaria.

Necesidad de armonía: es una tendencia a mantener un equilibrio en las relaciones interpersonales.

Comprensión: consiste en que las personas intentan entender y justificar a los demás, aunque estos actúen en contra de ellos y de sus intereses.

Represión emocional: es la inhibición inconsciente de la expresión de las emociones.

La represión emocional se ha planteado como el factor psicológico que predice un peor ajuste psicosocial en las personas que sufren cáncer, y que puede condicionar los resultados de los tratamientos médicos (Durá, et al., 2010).

La personalidad tipo $\mathrm{C}$ no es exclusiva de pacientes oncológicos. Se le relaciona con otras enfermedades crónicas, como la diabetes, cáncer de pulmón, enfermedades cardiovasculares, problemas gastrointestinales y endócrinos, asma y dolor crónico benigno (Anarte et al., 2000; Vinaccia, 2003). Sin embargo, es precisamente en estudios de la relación de la personalidad C con el cáncer de mama donde se han establecido dos líneas de investigación sobre la personalidad tipo C. En la primera se le considera como una característica premórbida para el desarrollo del cáncer. En éste sentido, diversos estudios encuentran que los pacientes presentan puntuaciones altas en las escalas de control emocional y comprensión (GrossarthMaticek, Bastiaans, \& Kanazir, 1985; López et al., 2002). En la segunda se considera un 
estilo de afrontamiento ante la enfermedad y en investigaciones con este enfoque se han encontrado altas puntuaciones en las escalas de necesidad de armonía y racionalidad (FernándezBallesteros, Ruiz, \& Garde, 1998; Temoshok \& Dreher, 1992; Vinaccia, 2003).

Estas diferencias de interpretación del constructo de personalidad tipo $\mathrm{C}$ generan la necesidad de continuar con investigaciones que contribuyan a dilucidar su naturaleza y su relación con el cáncer de mama y otras patologías. Para ello es conveniente contar con instrumentos válidos y confiables en la población específica con la que se pretenda trabajar. Hasta la fecha, gran parte de los estudios realizados han utilizado el Cuestionario de Personalidad tipo C (PCTC), elaborado por López et al. (1998, 2002). En el medio latinoamericano, existe una adaptación a la población colombiana llevada a cabo por Amaya, Gordillo y Restrepo-Forero (2006). Sin embargo, el uso de estas versiones del cuestionario en una población para la cual no fueron realizadas inicialmente se enfrenta con dificultades psicométricas, teóricas y prácticas.

En la literatura psicométrica se ha documentado que no existen garantías de que la estructura factorial encontrada en otras poblaciones se reproduzca en aquella en la que se trabaja (Prieto \& Delgado, 2010), y las diferencias en la estructura de la escala PCTC encontradas por López et al., (2002), y por Amaya et al. (2006), así lo confirman. Desde las perspectivas psicométrica y teórica, el hecho de que la estructura del cuestionario varíe de una población a otra hace inválidas las inferencias que se hagan con las puntuaciones del instrumento (Muñiz, 2010; Prieto \& Delgado, 2010). Igualmente, las fuentes de error aleatorio que inevitablemente existen en la aplicación de las escalas siempre pueden variar de una población a otra, e incluso de una aplicación a otra, lo cual hace necesario determinar la confiabilidad de la medición cada vez que esta se realice (Cook \& Beckman, 2006; Muñiz, 2010).

Desde el punto de vista teórico, también es indispensable documentar las diferencias en la estructura factorial en poblaciones distintas, ya que una o varias de las dimensiones propuestas por el enfoque teórico en el que se basa la escala pueden desaparecer o fusionarse en una sola, como en el trabajo ya citado de Amaya et al. (2006). Estas diferencias pueden dar origen a dificultades o errores de interpretación de los puntajes obtenidos.

Finalmente, desde el punto de vista práctico, el uso de una escala no adaptada a la población objetivo puede afectar la interpretación de los reactivos por las personas que responden, generando confusión y desconfianza. Esta situación atenta contra la validez y la confiabilidad de la aplicación (Oluwatayo, 2012). En los trabajos preliminares para el presente estudio, se pudo comprobar que diversos vocablos (de manera destacada, la palabra "enfado") de la escala original de López et al. (1998) produjeron grandes confusiones en los encuestado.

Además de lo anterior, en México solamente hemos encontrado un estudio en el que se determinó la relación entre la personalidad tipo C y el cáncer (Molina, Hernández \& Sarquis, 2009). Pero en dicho estudio no se realizó el trabajo de adaptación y validación del instrumento con población mexicana. Por todas estas razones, el objetivo del presente estudio es adaptar y validar el cuestionario PCTC en población mexicana.

\section{Método}

\section{Participantes}

El instrumento se aplicó a una muestra de 391 personas que participaron voluntariamente en el estudio, de ellas el $76.7 \%$ correspondía a mujeres y el $23.3 \%$ a hombres. Sus edades oscilaron entre los 18 y 70 años. Las aplicaciones se realizaron entre los meses de enero a septiembre del año 2013, en las ciudades de Chihuahua y Xalapa.

En Chihuahua se aplicaron 200 cuestionarios a población abierta de universidades privadas, alumnos de las carreras de psicología industrial, criminología y sistemas computacionales, además de un centro de atención y prevención psicológica. La muestra de Xalapa consta de 191 mujeres, usuarias de los sistemas de salud, 
a quienes se invitó a participar en el estudio mientras se encontraban en la sala de espera de 4 centros de salud diferentes ubicados en la misma ciudad.

\section{Instrumento}

Se utilizó el cuestionario de Personalidad Tipo C (PCTC) elaborado por López et al. (1998), y reestructurado en 2002, aplicable a personas sanas y enfermas. Consta de 26 ítems que se agrupan en 5 factores: control emocional, con 5 ítems; racionalidad, con 4; represión emocional, con 6 ítems; necesidad de armonía, con 6; y comprensión, con 6. Las respuestas a dichos ítems son dicotómicas, en formato sí o no; y se puntúan con 0 las respuestas de no y con 1 las respuestas sí (López et al., 2002).

\section{Procedimiento}

Se realizó una adaptación lingüística a los ítems del cuestionario por dos psicólogos conocedores del constructo, los cuales modificaron sucesivamente los reactivos para adaptarlos al modo de expresión lingüística de la población mexicana. Las modificaciones fueron piloteadas con una muestra inicial de diez pacientes, quienes sugirieron modificaciones adicionales para integrar una versión definitiva de los reactivos. De manera destacada, se rescató redacción impersonal de los reactivos tal como aparecen en el trabajo de López et al. (2002). Todos los participantes en el estudio tuvieron el auxilio permanente de los aplicadores de la prueba, en caso de que alguno de los términos de los reactivos fuera poco claro. Mediante este procedimiento se consiguió una adaptación satisfactoria de los reactivos, y las faltas de claridad reportadas fueron mínimas. Para llevar a cabo las aplicaciones, se capacitó a tres psicólogos clínicos en la ciudad de Chihuahua, y en la ciudad de Xalapa a una psicóloga, una enfermera psiquiátrica y una trabajadora social.

Una vez recopilados los datos, se procedió a hacer un análisis de poder discriminativo de los reactivos por medio del método de grupos contrastantes, así como un análisis de homogeneidad, verificando si las puntuaciones de cada reactivo correlacionaban con los totales de la escala completa y de cada factor. Posteriormente se llevó a cabo un análisis exploratorio para conocer la estructura factorial del cuestionario en población mexicana. Finalmente, se llevó a cabo un análisis factorial confirmatorio para comprobar si los elementos principales del constructo son los mismos que reportaron López et al. $(1998,2002)$ en población española. La confiabilidad para toda la escala y para cada uno de los factores encontrados se determinó mediante la fórmula de alfa de Cronbach.

\section{Análisis de datos}

Los análisis psicométricos, el análisis factorial exploratorio y la determinación de confiabilidad se hicieron con el programa estadístico IBM ${ }^{\mathrm{TM}}$ SSPS $^{\mathrm{TM}}$ 20. El análisis factorial confirmatorio se realizó con el programa AMOS SPSS ${ }^{\mathrm{TM}} 20$.

\section{Resultados}

Una vez integrada y depurada la base de datos, las puntuaciones de los reactivos fueron sometidas a la prueba de KolmogorovSmirnov. El procedimiento determinó que las puntuaciones no se distribuían normalmente. Por lo tanto, los análisis de poder discriminativo, de homogeneidad y factorial se llevaron a cabo por medio de procedimientos no paramétricos.

\section{Poder discriminativo y homogeneidad de los reactivos}

El análisis de poder discriminativo por el método de grupos contrastantes (Anastasi \& Urbina, 1998) demuestra que las puntuaciones del cuartil inferior de todos los reactivos es estadísticamente diferente de las puntuaciones del cuartil superior (prueba U de Mann-Whitney, $\mathrm{p}<0.001$ para todos los reactivos). El análisis de homogeneidad 
muestra que todos los reactivos presentan una adecuada correlación con su subescala correspondiente (correlación de Spearman, $r$ de 0.546 a 0.834 ), por lo cual no fue necesario descartar ningún reactivo en esta etapa del análisis.

\section{Medidas de adecuación muestral y análisis factorial exploratorio}

Con el propósito de determinar si la muestra empleada era adecuada para llevar a cabo el análisis factorial exploratorio, se realizaron las medidas de adecuación muestral pertinentes. La prueba de Kaiser-Meyer-Olkin dio un índice calculado de 0.762 , el cual se considera aceptable para llevar a cabo el análisis factorial (Beavers et al., 2013). La determinante de la matriz de correlaciones (0.002), y la prueba de esfericidad de Bartlett $(\mathrm{p}<0.001)$, descartan la posibilidad de que los reactivos no correlacionen entre sí y de que no sea posible extraer factores de la matriz de correlaciones (Beavers et al., 2013).

Dada la no normalidad de los datos, se realizó el análisis factorial por medio de la técnica de mínimos cuadrados no ponderados. Al principio se escogió un método de rotación oblicua (oblimín directo) para determinar si existían correlaciones significativas entre los factores obtenidos. Este primer análisis dio como resultado la presencia de 7 factores que correlacionaban muy poco entre sí y explicaban el $52.9 \%$ de la varianza, por lo cual se procedió a repetir el análisis utilizando un método ortogonal de rotación (varimax).

Al analizar la matriz de correlaciones obtenida, se hizo evidente que los últimos tres factores estaban representados solamente por un reactivo con un peso factorial apenas superior a 0.4 , y se repitió el análisis forzando una estructura de 4 factores. Esta nueva extracción dio lugar a 4 factores que explican el $42.28 \%$ de la varianza común (Tabla 1), lo cual se considera aceptable para un cuestionario de personalidad (Anastasi \& Urbina, 1998). El porcentaje de varianza explicada se refiere a la varianza común, que se encuentra libre de errores de medición (Beavers et al., 2013).

\section{TABLA 1}

Análisis factorial. Varianza total explicada por los 4 factores

\begin{tabular}{|c|c|c|c|c|c|c|c|c|c|}
\hline \multirow[t]{2}{*}{ conponente } & \multicolumn{3}{|c|}{ Anto rabies wiccules } & \multicolumn{3}{|c|}{ 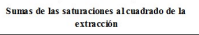 } & \multicolumn{3}{|c|}{ 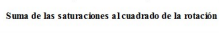 } \\
\hline & Tolat & 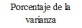 & 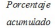 & Toala & $\begin{array}{l}\text { Porcerestip de } \\
\text { vatiana }\end{array}$ & 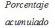 & Total & 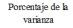 & 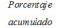 \\
\hline 1 & 4157 & 20.84 & 20.784 & 3.58 & 17901 & 17901 & 3.247 & 16.235 & 16235 \\
\hline 2 & 2.68 & 13.4 & 34.185 & 2222 & 11.108 & 29009 & 2.145 & 10726 & 26651 \\
\hline 3 & 1.897 & 9.486 & 43.67 & 1.377 & 6.885 & 35.84 & 1.648 & 8.241 & 35,202 \\
\hline & 1.849 & 9.244 & 52.914 & & 6.393 & 42887 & 1.417 & 7.064 & 42.837 \\
\hline
\end{tabular}

Fuente: elaboración propia

Los resultados del análisis factorial condujeron a eliminar 3 reactivos que no correlacionaron con ningún factor, y otros tres que formaron un factor sin relación con la teoría, con correlaciones negativas y significativas con el resto de los factores, y que dificultan la interpretación final de la escala. Esta eliminación produjo la estructura factorial rotada que se presenta en la tabla 2.

\section{TABLA 2}

Cargas factoriales de los reactivos del Cuestionario PCTC

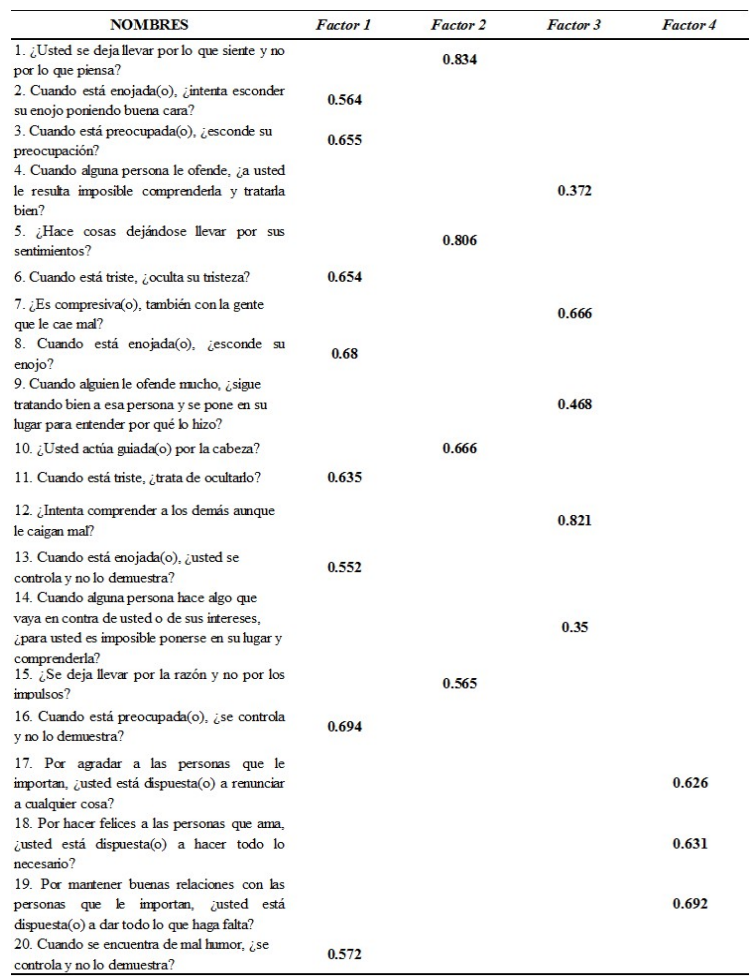

Fuente: elaboración propia. 


\section{Análisis factorial confirmatorio}

Para comprobar si la estructura factorial encontrada se ajusta a los datos y al enfoque teórico con el que está planteada, se llevó a cabo un análisis factorial confirmatorio con un modelo que plantea la existencia de 4 factores relacionados entre sí por un constructo global de segundo orden: la conducta o patrón tipo C (figura 1).

\section{Figura 1}

Modelo propuesto para el análisis factorial confirmatorio

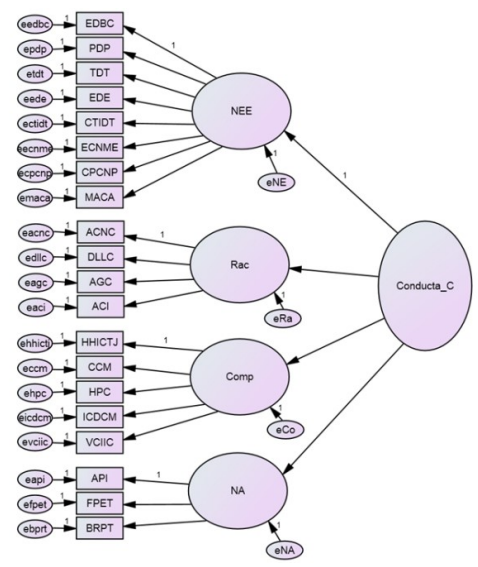

Notas: se describen solamente las variables no observables (factores). NEE $=$ No expresión emocional, $\mathrm{Rac}=$ Racionalidad, $\mathrm{Comp}=$ Comprensión, NA = Necesidad de armonía, Conducta_C $=$ Patrón de conducta tipo C. Fuente: elaboración propia.

Los resultados muestran que el factor general al que llamamos conducta tipo C explica 16\% de la variabilidad del factor no expresión emocional, $66 \%$ de la variabilidad del factor comprensión, $8 \%$ de la variabilidad de necesidad de armonía y $2 \%$ de variabilidad de la dimensión de racionalidad.

Como los índices de bondad de ajuste de este modelo no resultaban adecuados, se modificó el modelo eliminando el factor para el cual la varianza explicada era muy pequeña. Esto llevó al modelo que se ilustra en la figura 2, el cual muestra que los componentes fundamentales de la conducta tipo $\mathrm{C}$ en población mexicana son la no expresión emocional, con el $87 \%$ de la varianza explicada por el factor de segundo orden; la comprensión, del cual el patrón de personalidad tipo $\mathrm{C}$ explica el $6 \%$ de la varianza, y la necesidad de armonía, con el $2 \%$ de la varianza explicada. Los índices de ajuste para este modelo son excelentes, de acuerdo con criterios modernos (Byrne, 2012): TLI (TuckerLewis Index), GFI (Goodness of fit index) y CFI (Comparative Fit Index) son iguales a 1, mientras que RMR (Root Median Residual), RMSEA (Root median square error of aproximation) y el coeficiente CMIN/DF (mínimum discrepancy / degrees of freedom) tienen valores de 0 . Dado que los reactivos en realidad tienen una escala categórica y no continua, la estimación de los parámetros del modelo se confirmó utilizando el método de estimación bayesiana, obteniendo los mismos parámetros con una precisión mínima de 3 decimales. El valor del estadístico de Hoelter (396) mostró que el tamaño de la muestra era el adecuado para obtener una estimación confiable de los parámetros del modelo (Byrne, 2012).

\section{Figura 2}

Dimensiones fundamentales de la personalidad tipo C en población mexicana

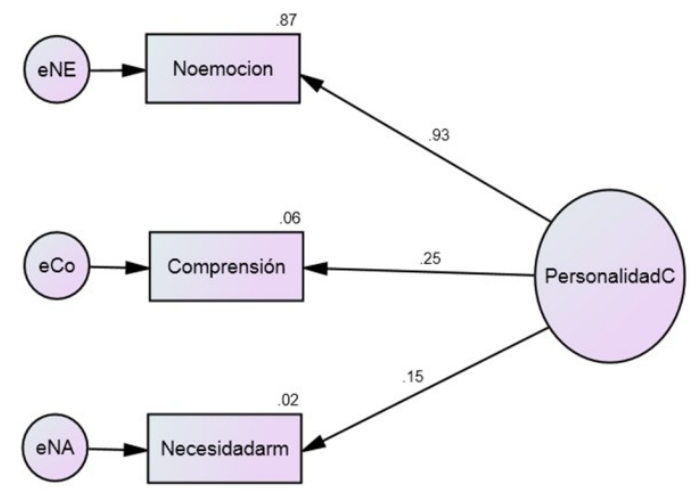

Notas: se reportan los coeficientes estandarizados. No emoción $=$ No expresión emocional, Necesidadarm $=$ Necesidad de armonía Fuente: elaboración propia.

\section{Diferencias en el modelo estructural entre subpoblaciones}

Cuando se compararon las dos subpoblaciones obtenidas de las ciudades de Xalapa y Chihuahua, se encontró que los datos obtenidos en Chihuahua (al norte de México) se ajustaban 
perfectamente al modelo estructural obtenido en el análisis general, con una muy pequeña disminución de varianza explicada para el factor de no expresión emocional (86\% vs. 87\%). Sin embargo, esta estructura no se ajustaba a lo encontrado en la ciudad de Xalapa (centro oriente de México). Los índices de modificación y el análisis de residuales contribuyeron a determinar que el factor de necesidad de armonía no era significativo en esta población. La eliminación de este factor lleva a un modelo en el cual, el constructo de personalidad tipo C explica el $88 \%$ de la varianza del factor no expresión emocional, y el 3\% del factor comprensión. En ambos modelos, los índices de ajuste CFI, TLI y GFI presentaron valores de 1; y los índices RMR, RMSEA y el cociente CMIN/DF tuvieron valores de 0. Los valores del estadístico de Hoelter (1193 para la primera subpoblación y 292 para la segunda) mostraron que el tamaño de cada submuestra fue apropiado.

\section{Análisis de confiabilidad}

La confiabilidad total de la escala se considera aceptable $(\alpha=0.757)$. La confiabilidad para cada una de las subescalas fue la siguiente: no expresión emocional, $\alpha=0.842$; racionalidad, $\alpha=0.809$; comprensión, $\alpha=0.645$ y necesidad de armonía, $\alpha=0.696$. Las confiabilidades obtenidas pueden ser consideradas como buenas para el caso de la escala total, y para las subescalas de racionalidad y no expresión emocional, y aceptables para las subescalas de comprensión y necesidad de armonía. La tabla 3 muestra la estructura final del cuestionario y los puntajes de confiabilidad para cada una de las subescalas.
TABLA 3

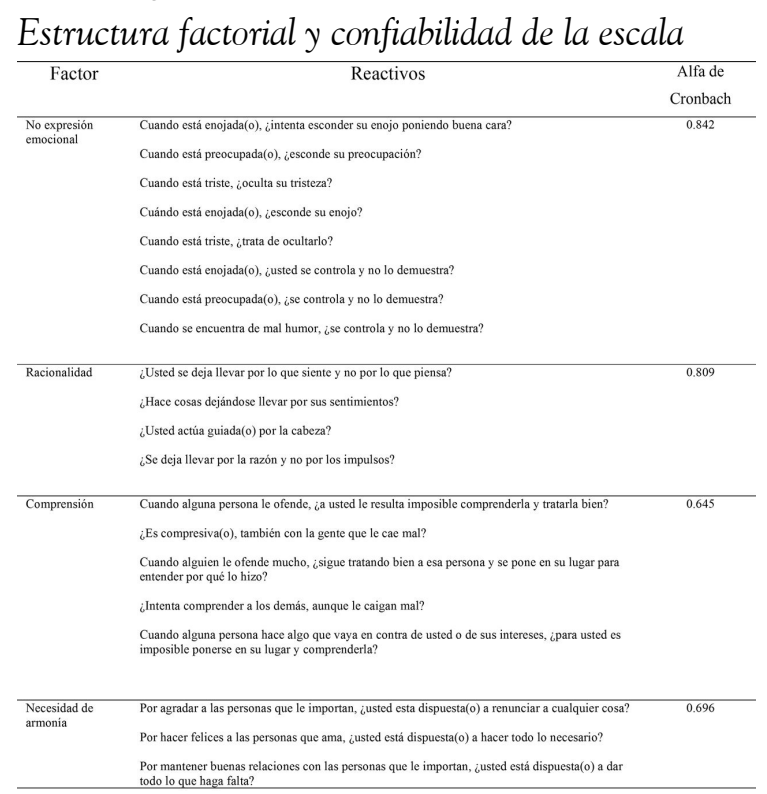

Fuente: elaboración propia

\section{Baremación}

Para situaciones diagnósticas, resulta útil obtener los criterios de baremación de la escala. Como se señaló anteriormente, los puntajes de la escala no se distribuyeron normalmente, y por esta razón se utilizó el criterio de dividir las puntuaciones de la población en terciles, y utilizar el puntaje correspondiente al límite inferior del tercil superior como punto de corte para la presencia de personalidad tipo C (Anastasi \& Urbina, 1998), el cual es igual a 12. De manera adicional, los puntajes límite del segundo tercil (8 para el límite inferior y 12 para el límite superior), pueden considerarse como indicadores de que la persona tiene rasgos de personalidad tipo C.

\section{Discusión}

Mediante el presente estudio se logró adaptar y validar en población mexicana el Cuestionario PCTC de López et al. (1998, 2002). Se obtuvo una escala compuesta por 20 reactivos de respuesta dicotómica agrupados en 4 factores o subescalas: factor 1: no expresión emocional, 8 reactivos; factor 2: racionalidad, 4 reactivos; factor 3: comprensión, 5 reactivos y factor 4: 
necesidad de armonía, 3 reactivos (Tabla 3 ). La varianza explicada por los 4 factores fue de $42.28 \%$, la cual se considera aceptable para una escala de personalidad (López et al. 2002; Amaya et al. 2006).

La estructura factorial obtenida es similar a la que se obtuvo en el estudio realizado por las investigadoras colombianas Amaya et al. (2006), por lo que los factores represión emocional y control emocional de la escala original se juntaron en un solo factor, denominado "no expresión emocional'. Este resultado no se vio alterado por la eliminación de tres reactivos adicionales con respecto a la versión colombiana (uno del factor comprensión y dos de control emocional), por lo que la estructura final de 4 factores se considera apropiada para la población mexicana. Los tres reactivos mencionados no correlacionaron con ningún factor, ni siquiera en pequeña proporción (la carga factorial máxima fue de 0.18), lo que mostró su inoperatividad en la población.

Los otros tres reactivos coinciden con los que se desecharon en la versión colombiana del cuestionario, y cuya redacción incluye términos como: "está dispuesta(o) a ceder solo hasta cierto límite". Las razones para eliminarlos fueron: 1) los reactivos formaron un factor único con cargas factoriales superiores a 0.6, con cargas factoriales negativas con los otros factores, y con correlación negativa de moderada a alta con el puntaje total de la escala (correlación de Spearman entre -0.45 y - 0.82), y; 2) analizando la redacción de estos reactivos, se consideró que, en población mexicana, parecen formar parte de un factor relacionado con la asertividad; constructo psicológico que no tiene relación con la personalidad tipo $\mathrm{C}$, y que ha sido definido como la capacidad de dejar en claro la posición personal con respecto a un tema concreto (Castanyer, 2012).

Los resultados del análisis factorial confirmatorio muestran diferencias con respecto a las dos versiones anteriores de la escala PCTC. Las autoras de la escala original encontraron como factores fundamentales de la personalidad tipo $\mathrm{C}$ a la represión emocional, el control emocional y la comprensión. En el modelo realizado en el presente estudio, se encontró que los factores de represión y control emocional se fusionan en uno solo, como lo observaron Amaya et al. (2006). Pero surgió como factor adicional la necesidad de armonía, que aunque tiene una muy pequeña proporción de varianza explicada, resulta crucial para el ajuste adecuado del modelo. El ajuste de este nuevo modelo de tres factores resultó tan exacto como el que encontraron las creadoras de la escala original.

Un aspecto novedoso del presente trabajo fue el análisis de la estructura factorial por subpoblaciones, que reveló que la estructura de la escala no solo puede presentar diferencias entre los países, sino entre distintas zonas dentro de un mismo país. Desde el punto de vista psicométrico y por las razones anotadas en la Introducción, esto no es sorprendente. La posibilidad de que exista funcionamiento diferencial de ítems siempre hace aconsejable que se realice el análisis de la equivalencia funcional de los reactivos (Byrne, 2012). En este caso particular, que se trataba de dos poblaciones de composición tan diferente, se hacía imprescindible la confirmación de la equivalencia funcional de los reactivos.

Entre las posibles explicaciones de las diferencias encontradas están, por un lado, las características propias de la población mexicana, particularmente aquellas que se relacionan con la comprensión del lenguaje utilizado para construir los ítems y, por otro, la manera de conducir el análisis factorial confirmatorio. Existen muchas evidencias de que las diferencias étnicas y culturales pueden condicionar la estructura de un constructo o una escala, en una población dada (Demianczyk, Jenkins, Henson, \& Conner, 2014). Lo mismo puede ocurrir con la manera de presentación de los reactivos (Anastasi \& Urbina, 1998). Estas aseveraciones se ven respaldadas por la confusión que generaron en los respondientes mexicanos algunos vocablos de la versión original, y el formato de presentación personalizado para los reactivos.

En lo que se refiere al análisis factorial confirmatorio, se trata de una técnica concebida inicialmente para ser utilizada con datos que cumplieran los supuestos de continuidad y 
normalidad multivariante (Kline, 2011). López et al. (2002) y Amaya et al. (2006) reportaron haber utilizado el método de extracción conocido como Mínimos Cuadrados Generalizados en sus análisis, pero la investigación reciente muestra que los métodos de estimación diferentes al método convencional de Máxima Verosimilitud tienen serios inconvenientes (Byrne, 2012). Por esta razón, en el presente estudio se utilizó el método de Máxima Verosimilitud, y los resultados obtenidos se comprobaron con el método de estimación bayesiana, que resulta particularmente útil para variables categóricas dicotómicas (Arbuckle, 2010; Byrne, 2012). Ambos métodos estimaron los mismos valores para los pesos de regresión y las varianzas explicadas, con una precisión de al menos 3 decimales, tanto en la población general como en las dos subpoblaciones.

Las diferencias entre los resultados de diversos estudios en los que se ha utilizado la escala PCTC han llevado a algunos autores a considerar que la personalidad tipo $\mathrm{C}$ no es en realidad un constructo unitario (López et al., 2002; Sebastián et al., 2009). Entre los argumentos a favor de esta interpretación se cuenta el hecho de que la escala PCTC fue construida a partir de cinco cuestionarios diferentes, y es posible que los cuestionarios midan aspectos diversos, tanto los relativos a un patrón de personalidad como a un estilo de afrontamiento; dependiendo del tipo de población con que se trabaje y del diseño del estudio. Sin embargo, los resultados del presente estudio muestran que la estructura del constructo puede variar sustancialmente entre las poblaciones, y el uso indiscriminado de la escala sin la necesaria confirmación de su estructura factorial en cada población, puede influir en la interpretación de los resultados y del enfoque teórico que los sustenta (Prieto \& Delgado, 2010). Por estas razones, los estudios futuros que busquen aclarar la naturaleza del constructo de personalidad tipo C deberían tener, además del diseño adecuado, una sólida fundamentación psicométrica basada en la investigación de la estructura del constructo en la población de interés.
Finalmente, entre las posibles críticas que pueden hacerse al presente estudio se encuentra el tamaño de la muestra, que es sustancialmente menor que la de los estudios anteriores (391, vs. 618 del primer estudio de López et al., 2002, y 1.121 en el trabajo de Amaya et al., 2006). Sin embargo, la muestra empleada fue heterogénea, recolectada en dos poblaciones mexicanas representativas y en cantidades suficientes y con procedimientos de análisis adecuados para llevar a cabo estimaciones confiables, según las guías modernas de análisis factorial exploratorio (Beavers et al., 2013; Gaskin \& Happell, 2014; Lloret-Segura, Ferreres-Traver, Hernández-Baeza \& Tomás-Marco 2014). Es bien conocido el hecho de que la confiabilidad de una escala depende de la heterogeneidad de la muestra empleada (Muñiz, 2010; Prieto \& Delgado, 2010), y los coeficientes obtenidos en el presente trabajo son por lo menos tan buenos como los encontrados por López et al., 2002 y Amaya et al., 2006, tanto en los análisis exploratorios como en los confirmatorios.

En conclusión, los resultados del presente trabajo permitieron obtener una versión de la escala PCTC válida, confiable y adaptada para su aplicación en población mexicana, a la vez que advierten del hecho de que en diferentes subpoblaciones, es necesario realizar el trabajo de validación necesario para interpretar los puntajes de manera apropiada. No es posible utilizar indiscriminadamente la información psicométrica reportada en la literatura. Los investigadores deben garantizar que el instrumento utilizado se ajuste a las características que el constructo de interés presenta en la población con la que trabajan, tal como se ha reportado en tantas ocasiones en la literatura psicométrica (Prieto \& Delgado, 2010).

\section{Referencias}

Amaya, A.T., Gordillo, L., \& Restrepo-Forero, M. (2006). Validación del cuestionario PCTC en población colombiana. Avances en medición, 4, 91-122. 
Amaya, A.T., \& Parra, J.E. (2010). Evaluación del patrón de conducta tipo $\mathrm{C}$ y su relación con el control emocional en pacientes oncológicos. Vanguardia Psicológica, 1(1), 70-89.

Anarte, M.T, López, M., Ramírez, M., \& Zarazaga, R. (2000). Evaluación del patrón de conducta tipo $\mathrm{C}$ en pacientes crónicos. Anales de Psicología, 16(2), 133-141.

Anastasi, A., \& Urbina, S. (1998). Test psicológicos (7a Ed.). México: Pearson Prentice Hall.

Arbuckle, J. (2010). Amos ${ }^{\text {TM }}$. User's Guide. IBM SPSS ${ }^{\circledR}$ Amos Development Corporation.

Beavers, A., Lounsburyn, J., Richards, J., Huck, S., Skolits, G., \& Esquivel, S. (2013). Practical considerations for using exploratory factor analysis in educational research. PARE, 18(6), 1-13.

Byrne, B.M. (2012). Structural equation modeling with AMOS. Basic concepts, applications and programming (2nd Ed.). New York, United States: Routledge.

Castanyer, O. (2012). La asertividad: expresión de una sana autoestima (3a Ed.). Barcelona, España: Desclee de Brower.

Cook. D.A., \& Beckman, T.J. (2006). Current Concepts in Validity and Reliability for Psychometric Instruments: Theory and Application. The American Journal of Medicine, 119, (166.e7-166.e16). doi: http:/ /dx.doi.org/10.1016/j.amjmed.2005.10.036

Durá, E., Pérez, S., Ferrando, M., Murgui, S., \& Martínez, P. (2010). Emotional suppression \& breastcancer: validation research on the Spanish adaptation of the courtauld emotional control scale. The spanish journal of psychology, 13(1), 406-417.

Demianczyk, A.C., Jenkins A.L., Henson, J.M., \& Conner, B.T. (2014) Psychometric evaluation and revision of Carver and White's BIS/BAS scales in a diverse sample of Young adults. J Pers Assess, 96(5), 485-94. doi: $10.1080 / 00223891.2013 .870570$

Fernández Ballesteros, R., Ruiz, M. A. \& Garde, S. (1998). Emotional expression in healthy women and those with breast cancer. British Journal of Health Psychology, 3, 41-50. doi: http://dx.doi.org/10.1111/j.2044-8287. 1998.tb00554.x

Gaskin, C.J., \& Happell, B. (2014). On exploratory factor analysis: A review of recent evidence, an assessment of current practice, and recommendations for future use. International Journal of Nursing Studies, 51, 511-521.doi: http://dx.doi.org/10.1016/ j.ijnurstu.2013.10.005

Grossarth-Maticek, R., Bastiaans, J., \& Kanazir, D. (1985). Psychosocial factors as strong predictors of mortality from cancer, ischaemic heart disease and stroke: The Yugoslav prospective study. J Psychosom Res, 29(2),167-76. doi: http://dx.doi.org/10.101 6/0022-3999(85)90038-8

Kline, R.B. (2011). Principles and practice of Structural equation modeling (3rd Ed.). New York, United States: The Guilford Press.

Lloret-Segura, S., Ferreres-Traver, A., Hernández-Baeza., A. \& Tomás-Marco, I. (2014). El análisis factorial exploratorio de los ítems: una guía práctica revisada y actualizada. Anales de Psicología, 30, 1151-1169. doi: http://dx.doi.org/10.6018/ analesps.30.3.199361

López. M., Esteve, R., Ramírez, C., \& Anarte, M. (1998). Dimensionalización del constructo de personalidad tipo C. Psykhe, 7(1), 3-12.

López, M., Ramírez, C., Esteve, R. \& Anarte, M. (2002). El constructo de personalidad tipo C una contribución a su definición a partir de datos empíricos. Psicología conductual, 10, 229-249.

Molina, V., Hernández, L. \& Sarquís, A. (2009). Patrón de personalidad tipo $\mathrm{C}$ y su relación con cáncer de mama y cérvico-uterino. Revista Electrónica de Psicología Iztacala, 12(4), 171-187.

Morris, T., \& Greer, S. (1980). A "Type C" for cancer? Low trait anxiety in the pathogenesis of breast cancer. Cancer Detection and Prevention, 3, Abstract No. 102.

Muñiz, J. (2010). Las teorías de los test: teoría clásica y teoría de respuesta a los ítems. Papeles del psicólogo, 31 (1), 57-66. 
Oluwatayo, J. A. (2012). Validity and Reliability Issues in Educational Research. Journal of Educational and Social Research, 2(2), 391-400.

Prieto, G., \& Delgado, A.R. (2010). Fiabilidad y Validez. Papeles del Psicólogo, 31 (1), 67-74.

Sebastián, J., León, M., \& Hospital, A. (2009). Variables psicosociales y cáncer de mama: un estudio cuasi prospectivo de la personalidad tipo C. Latinoamericana de psicología, 41 (3), 461-479.

Temoshok, L., \& Dreher, H. (1992). The Type C Connection: The Behavioral Links to Cancer and Your Health. United States: Random House.

Vinaccia, S. (2003). El patrón de conducta tipo $\mathrm{C}$ en pacientes con enfermedades crónicas. Colombiana de psiquiatría, XXXII (1), 161-168.

\section{Notas}

* Artículo de investigación. 